Canadian Oncology

Nursing Journal

Revue canadienne

de soins infirmiers

en oncologie

Volume 29, Issue 3 • Summer 2019

elSSN: 2368-8076 


\title{
Expériences des soins de soutien des femmes atteintes de cancer du sein vivant en milieu rural : étude qualitative et description interprétative
}

\author{
par Joanne Loughery et Roberta L. Woodgate
}

\section{RÉSUMÉ}

Au Canada, le cancer du sein est le type de cancer le plus fréquent chez les femmes. Malgré tout, la recherche s'est peu intéressée aux besoins en matière de soins de soutien des femmes des régions rurales aux prises avec cette maladie. L'objectif de la présente étude qualitative est donc de mieux comprendre les expériences de soins de soutien des Manitobaines qui vivent en milieu rural et qui doivent subir des traitements pour un cancer du sein invasif. Le phénomène a été exploré à l'aide d'un devis qualitatif, descriptif et interprétatif. Vingt femmes de quatre régies régionales de la santé ont participé à l'étude. Des entrevues semi-structurées conduites en personne ont permis de recueillir les données, qui ont par la suite été soumises à une analyse qualitative interprétative. Les résultats obtenus brossent un portrait holistique des expériences vécues par les femmes atteintes de cancer du sein qui reflète à la fois les aspects positifs et négatifs de la ruralité. Les recommandations pragmatiques, formulées à partir des résultats de l'étude, orienteront la pratique infirmière et la prestation des services.

Mots-clés : cancer du sein, soins de soutien, expériences, ruralité, étude qualitative, description interprétative

L e cancer est une maladie dévastatrice, quel que soit le lieu - de résidence de la personne qui en est atteinte. Au Canada, le cancer du sein est prédominant chez les femmes; chaque année, elles sont environ 26300 à en recevoir le diagnostic (Société canadienne du cancer [SCC], 2017). Un Canadien sur cinq vit par ailleurs en région rurale, ce qui correspond à 18,9\% de la population (Statistique Canada, 2015). Malgré tout, la recherche canadienne sur l'expérience d'avoir un cancer du sein en milieu rural est limitée : deux études seulement figurent dans la littérature à cet égard (Gray, James,

\section{AU SUJET DES AUTEURS}
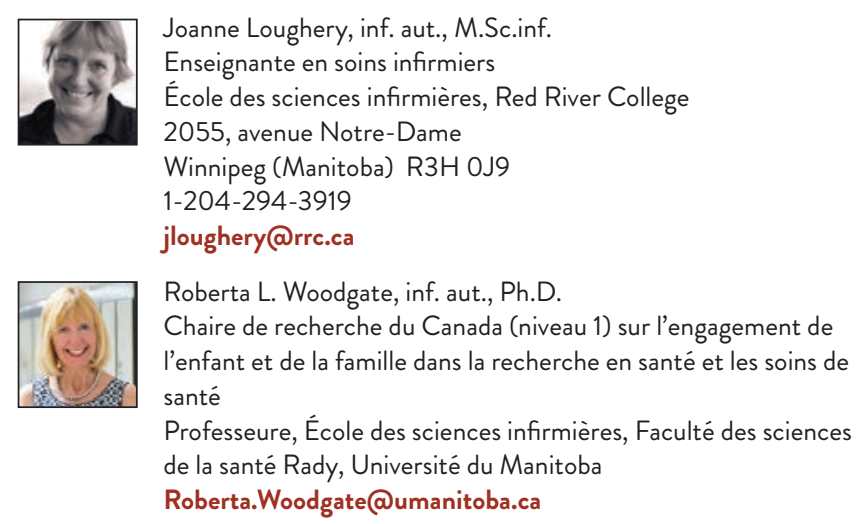

DOI: $10.5737 / 23688076293177183$
Manthorne, Gould et Fitch, 2004; Fitch et al., 2003). Avec l'incidence croissante du cancer du sein au pays, ce sujet demeure prépondérant. En outre, comme l'accès aux services et aux spécialistes en oncologie est restreint dans les régions rurales, les patients doivent inévitablement voyager beaucoup et se reloger. Nous avons choisi de réaliser une étude qualitative pour donner la parole aux Manitobaines des régions rurales atteintes de cancer du sein et jeter les fondements théoriques des possibilités d'intervention et de changement qui méritent considération.

\section{CONTEXTE}

$\mathrm{Au}$ Manitoba, environ 860 femmes reçoivent un diagnostic de cancer du sein chaque année (SCC, 2017); dans $80 \%$ des cas, il s'agit de carcinomes canalaires infiltrants. Le traitement recommandé est donc multimodal, combinant chirurgie, chimiothérapie et thérapie biologique, radiothérapie et hormonothérapie à long terme (Crane-Okada et Loney, 2007; National Comprehensive Cancer Network [NCCN], 2004). L'expérience est cependant plus complexe pour les femmes vivant en milieu rural, vu la difficulté d'accès aux services d'oncologie et la fragmentation des soins de santé en région. Au Manitoba, des soins du cancer spécialisés sont offerts dans la capitale provinciale; les patients peuvent aussi bénéficier de services d'oncologie multidisciplinaires, de chimiothérapie, de radiothérapie et de certaines interventions chirurgicales au Western Cancer Center, situé à Brandon, dans la deuxième ville en importance du Manitoba; quinze programmes communautaires de lutte contre le cancer en milieu rural s'occupent d'offrir de la chimiothérapie ainsi que la prise en charge des symptômes et des soins de suivi, et des programmes de navigation, d'oncologie psychosociale et de soins de première ligne en oncologie sont en place dans chaque régie régionale de la santé (Action Cancer Manitoba [ACM], 2000; 2017). Malgré tout, les patientes n'ont pas le choix de se rendre dans un centre urbain, car la chirurgie, la reconstruction mammaire et la radiothérapie ne sont pas accessibles dans la plupart des régions rurales.

Le Cadre de soins de soutien est un outil utilisé par les professionnels de la santé pour conceptualiser les besoins des patients aux prises avec le cancer (Fitch, 2009). Le cancer est un événement imprévu de la vie qui peut entraîner des sentiments d'anxiété et de vulnérabilité lorsque les besoins de base de la personne ne sont pas comblés, qu'ils soient physiques, sociaux, psychologiques, émotionnels, spirituels, informationnels ou pratiques. Et chaque fois que le traitement gagne en complexité, ces sphères de la vie se compliquent elles aussi (Fitch, 2009). Le Cadre de soins de soutien a servi de guide au 
processus de recherche de la présente étude.

Une revue de littérature a servi de base pour explorer le phénomène des soins de soutien et les expériences des femmes vivant en milieu rural qui sont atteintes de cancer du sein. La première étape a été de faire un survol des soins de cancer pour toute la trajectoire de la maladie, ce qui a fait ressortir combien chaque étape présentait des problèmes associés au traitement et défis particuliers (Crane-Okada et Loney, 2007; Fitch, 2009; NCCN, 2004).

Ensuite, l'exploration s'est tournée vers les besoins de toutes les femmes atteintes de cancer du sein. Chaque domaine du Cadre de soins de soutien regroupe des besoins différents, sans compter que la réaction à la maladie et au traitement n'est jamais exactement la même d'une personne à l'autre. Toutefois, dans le cas du cancer du sein et de l'expérience des patientes vivant en milieu rural, certaines tendances se dégagent. La littérature aborde quelques-unes des difficultés liées à la ruralité : l'exacerbation des symptômes physiques à cause des déplacements; l'accès limité aux médecins de famille; l'éloignement des spécialistes en oncologie; les perturbations sociales liées au relogement; le manque d'intimité dans les collectivités rurales; les frais supplémentaires que les patients doivent payer de leur poche pour le transport et le logement; les décisions thérapeutiques prises en fonction de l'incapacité à voyager (Fitch et al., 2003; Gray et al., 2004; Loughery et Woodgate, 2015; Matthews, West et Buehler, 2009; Pesut, Robinson, Bottorff, Flyes et Broughton, 2010). Toutefois, la vie à la campagne présente aussi de nombreux aspects positifs, mentionnés dans la littérature, par exemple : l'extension $\mathrm{du}$ soutien et les liens étroits entre les membres de la communauté; la résilience et la force de caractère, gages d'une adaptation efficace; la beauté et la tranquillité de l'environnement; la proximité de la congrégation religieuse d'appartenance (Compas et Luecken, 2002; Pesut et al., 2010; Rogers-Clark, 2002; Winter et Lee, 2010).

Enfin, les autres modèles de soins oncologiques ont été explorés selon une perspective nationale et internationale. De toute évidence, la décentralisation des soins du cancer vers les régions rurales améliorerait les services pour tous les patients, mais il y a tout de même place à l'amélioration (Adam et al., 2009; Carlow, 2000; Clinical Oncology Society of Australia, 2006; National Institute for Health and Clinical Excellence, 2004). Le présent projet de recherche a donc été lancé en tenant compte de tous ces éléments.

\section{BUT}

Le principal objectif de l'étude qualitative était de mieux comprendre l'expérience des soins de soutien des femmes adultes des régions rurales du Manitoba qui devaient subir un traitement pour un cancer du sein invasif. Les résultats obtenus couvrent toutes les étapes de la maladie, du dépistage à la fin du traitement, et ont permis de générer différentes recommandations.

\section{MÉTHODOLOGIE}

La description interprétative, modèle choisi pour la présente étude, permet d'investiguer qualitativement un phénomène clinique (Thorne, 2008). Cette méthodologie a servi de cadre pour comprendre l'expérience découlant de la maladie, de même que le rôle des infirmières et leur influence sur la vie des patients cancéreux. La description interprétative est ancrée dans l'orientation naturaliste, qui respecte les expériences individuelles tout en laissant l'espace nécessaire aux réalités communes. Ces réalités communes servent de fondement pour générer une description interprétative apte à orienter la pratique (Thorne, 2008). L'étude a été approuvée par le comité d'éthique de la recherche en sciences infirmières, quatre régies de la santé et Action Cancer Manitoba.

Les critères d'inclusion étaient les suivants : 1) femmes adultes capables de parler, lire et écrire l'anglais et ne souffrant d'aucun trouble cognitif; 2) diagnostic de cancer du sein invasif suivi d'une ronde de traitement multimodal dans les trois dernières années; 3) femmes résidant dans les régions rurales ou nordiques du Manitoba. Étaient exclues de l'étude les femmes qui avaient un cancer de stade IV, qui avaient connu une récidive du cancer ou qui souffraient d'une maladie évolutive, car les plans de traitement sont différents dans ces cas. Pour bien capturer l'essence de l'expérience vécue en cas de cancer du sein en milieu rural et en dégager les caractéristiques communes, nous avons employé un échantillonnage intentionnel (Streubert et Carpenter, 2011). Les patientes avaient le choix du milieu naturaliste où serait réalisée l'entrevue, ce qui a suscité des déplacements dans la province totalisant plus de $10000 \mathrm{~km}$.

Les données ont été recueillies par des entrevues en personne, l'administration de questionnaires démographiques, la prise de notes d'observation et la tenue de journaux. Vingt entretiens ont été réalisés pendant la première vague d'entrevues et douze dans la deuxième. Les auteures ont alors constaté que les données se répétaient et qu'aucune nouvelle information ne ressortait de la deuxième série d'entrevues (Streubert et Carpenter, 2011). Les huit derniers entretiens de la seconde vague n'ont donc pas eu lieu.

Les réponses à ces entrevues semi-structurées non directives ont été enregistrées et retranscrites textuellement. Toutes les entrevues ont été codées, puis examinées sous la loupe d'une analyse de contenu dirigée. Cette approche a permis de valider le cadre théorique en établissant des relations entre les données codées (Zhang et Wildemuth, 2009). Les deux auteures ont revu les données brutes à plusieurs reprises pour en extraire des descriptions interprétatives. Puis, par déduction, elles ont classé les données en fonction des catégories décrites dans le Cadre. À la fin de l'étude, chacune des participantes a reçu le sommaire des résultats, ainsi qu'une invitation à apporter toute clarification requise. L'engagement continu auprès des participantes, la vérification par les membres, la révision par les pairs, le débreffage et la consignation détaillée de la piste de vérification ont tous contribué à la rigueur méthodologique du travail (Streubert et Carpenter, 2011). 


\section{RÉSULTATS DÉMOGRAPHIQUES ET QUALITATIFS}

\section{Résultats démographiques}

Les données démographiques comprennent les descriptions des participantes, l'information sur les modalités de traitement, ainsi que les renseignements sur la région de résidence et les déplacements. Les données démographiques ont été recueillies à l'aide de questionnaires et de l'analyse de chaque entrevue.

Description des participantes. Les quatre régies régionales de la santé de la province étaient représentées par cinq femmes chacune. Lâge des participantes variait entre 34 et 85 ans, la majorité étant âgée entre 46 et 65 ans. Onze d'entre elles étaient mariées; les autres étaient célibataires, veuves ou séparées. Six femmes vivaient seules, les autres avec leur conjoint, leurs enfants adultes, leurs enfants ou leurs petits-enfants. Dix femmes occupaient un emploi au moment du diagnostic et cinq ont travaillé à divers moments pendant leur traitement. Elles exerçaient leur métier dans différents secteurs, notamment dans le domaine de la santé, de la vente au détail, dans un bureau ou une entreprise à domicile. La moitié des femmes avaient obtenu un diplôme d'études postsecondaires et les autres avaient poursuivi leur scolarité jusqu'en $12^{\mathrm{e}}$ année ou moins. Elles étaient majoritairement de race blanche.

Données démographiques des modalités de traitement. Dix femmes ont subi une lumpectomie et les autres une mastectomie unilatérale ou bilatérale avec, parfois, reconstruction mammaire ou pose d'implant. Toutes les femmes ont dû recevoir de 4 à 8 cycles de chimiothérapie, et sept d'entre elles ont été sous traitement biologique pendant une année supplémentaire. Toutes les femmes ont subi une radiothérapie du champ atteint et seize d'entre elles ont commencé l'hormonothérapie.

Données démographiques sur les régions et les déplacements. Onze femmes vivaient dans une ville de 7500 habitants ou plus (ou à proximité), les autres dans une ville de moins de 7500 habitants (ou à proximité). Six femmes avaient accès à un programme communautaire de lutte contre le cancer dans leur collectivité et quatre devaient se déplacer pour obtenir des services de chimiothérapie. Pour se rendre à Winnipeg, ces dernières parcouraient entre 62 et $1100 \mathrm{~km}$; celles qui allaient à Brandon faisaient entre 15 et $182 \mathrm{~km}$. Dans l'une des régions, les patientes pouvaient se rendre à Brandon pour obtenir des services supplémentaires, notamment la chirurgie, la chimiothérapie et la radiothérapie. Elles voyageaient principalement par voie terrestre, mais quatre participantes du nord de la province prenaient l'avion et recevaient une indemnisation du Programme de transport des malades du Nord. Dixsept femmes avaient été opérées à Winnipeg et les autres, à Brandon. Dix-huit participantes avaient reçu leur chimiothérapie grâce à un programme communautaire de lutte contre le cancer; les autres se rendaient à Winnipeg. Seize femmes avaient reçu de la radiothérapie à Winnipeg, et les autres, à Brandon.

\section{Résultats qualitatifs sur les soins de soutien}

Les résultats ont été catégorisés en fonction des domaines du Cadre sur les soins de soutien (Fitch, 2009). Les expériences les plus courantes sont décrites pour chaque domaine sans préciser les variations individuelles.

Domaine physique. Le domaine physique était très exigeant. Les effets secondaires variaient selon le mode de traitement, la chimiothérapie étant la plus éprouvante. De plus, la ruralité était associée à certaines difficultés physiques. À l'étape du diagnostic, certains éléments incontrôlables, liés au programme de mammographie mobile notamment, entraînaient des délais, par exemple les conditions de conduite hivernale. Pendant le traitement, les déplacements demeuraient pénibles, car ils aggravaient les symptômes physiques tels que la fatigue, la douleur ou les réactions cutanées. De plus, la sécurité pendant le trajet soulevait des inquiétudes, notamment à cause des symptômes comme la fatigue accablante, le « brouillard de la chimio » et l'impossibilité de porter la ceinture de sécurité après la chirurgie. L’une des participantes, qui conduisait tous les jours pour se rendre à ses traitements de radiothérapie, a mentionné la sécurité sur la route comme grande source d'inquiétude.

Mais vous savez, à cause du brouillard mental de la chimio et la fatigue causée par la radiothérapie, c'est difficile de réfléchir comme il faut. Je n'aurais pas dû conduire.

Pour les participantes, l'accès à des professionnels de la santé qualifiés expérimentés dans des établissements non voués aux soins du cancer ou dans les services d'urgence, limités dans les collectivités rurales, afin de trouver réponse à leurs besoins physiques complexes constituait également une préoccupation. Le fardeau du transport et des soins nécessaires pour satisfaire ces besoins physiques complexes en était d'autant plus lourd pour la famille ou les amis. Toutefois, les participantes ont mentionné le rôle positif des programmes de soins du cancer en milieu rural et des soins à domicile, qui avaient aidé bon nombre d'entre elles à supporter les difficultés physiques.

Domaine informationnel. L'information émanait de différents professionnels de la santé : médecins de famille, spécialistes en oncologie, personnel hospitalier, infirmières travaillant en milieu urbain et rural, infirmières pivots et autres professionnels interdisciplinaires. Plusieurs facteurs influençaient la capacité à assimiler l'information, notamment l'intensification des craintes, les longues journées de voyage, l'horaire de rendez-vous chargé, la complexité de l'information ou l'absence d'un accompagnateur disponible pour assister au rendez-vous. Une participante de cinquante ans, qui vivait loin d'un centre urbain, a mentionné les multiples rendez-vous avec des spécialistes auxquels elle devait assister en un court laps de temps. Elle s'est sentie obligée de prendre rapidement une décision par rapport à son traitement, qu'elle a fini par regretter.

Tout s'est passé si vite et je me suis mise à penser à ce qui pourrait arriver... si je n'ai pas de seins et que tout se passe si vite et que je n'ai pas de reconstruction... j'ai dî prendre une décision sur-le-champ pendant la discussion et tout allait juste trop vite... 
L'accès aux médecins de famille semble être inégal dans la province et la communication entre les établissements urbains et ruraux comporte sa part de défi. En outre, il appert que les fournisseurs de soins ou le personnel hospitalier en milieu rural ne possèdent qu'une connaissance limitée des problèmes liés au cancer, ce qui constitue un casse-tête supplémentaire. Les infirmières et le personnel des établissements ruraux de soins du cancer jouaient un rôle capital pour répondre aux besoins informationnels des femmes, mais faute d'espace ou de lieux adéquats, il leur était difficile de discuter en toute confidentialité. L'accès aux infirmières pivots ou à des professionnels de la santé interdisciplinaires était limité, même s'il aurait été fort à propos. Beaucoup d'autres ressources informationnelles ou services de soutien ont été utilisés, chacun présentant des avantages et des inconvénients.

Domaine social. Les participantes ont mentionné différents types de réseaux de soutien social, notamment la famille immédiate ou élargie, les amis, les membres de la communauté, auxquels elles avaient eu recours à différents moments de l'expérience du cancer. L'importance des réseaux de soutien variait aussi selon la situation familiale ou la présence de membres de la famille ou d'amis vivant dans la collectivité. Le célibat avait ses bons et ses mauvais côtés, mais comportait son lot de défis sur le plan pratique. De plus, les fournisseurs de soins de santé, et particulièrement les infirmières en milieu rural, étaient une source de soutien importante pour les femmes, qui en parlaient en termes positifs.

Il était évident que les participantes aimaient vivre en région rurale. Elles décrivaient le fort sentiment d'appartenance à la communauté et la tranquillité. Mais la vie rurale créait aussi son lot d'obstacles, comme le manque d'intimité, les intrusions dans la vie privée ou les difficultés de communication, ce qui causait souvent un sentiment d'isolement. L'une des participantes, qui habitait depuis toujours dans un petit village, voyait son diagnostic comme une expérience très personnelle, ce qui l'a poussée à s'isoler des autres membres de la communauté pendant toute la durée de son traitement. $\mathrm{Au}$ moment de l'entrevue, un an après le traitement, elle venait tout juste de recommencer à sortir.

Oui, parce que tout le monde sait tout sur tout le monde.

C'est comme ça. Lorsqu'on tient à sa vie privée, ce n'est franchement pas évident... et, généralement, tout finit par se savoir et la nouvelle se répand comme une traînée de poudre.

L'isolement pouvait aussi être causé par la nécessité de se reloger pour le traitement, les difficultés physiques inhérentes à la maladie et le fait d'éviter les endroits publics ou les interactions sociales. Plusieurs femmes ont dit que des gens qu'elles ne connaissaient pas ou qui n'étaient que de vagues connaissances leur avaient posé des questions très personnelles sur leur maladie ou avaient fait des commentaires déplacés ou gênants. Les participantes ont mentionné d'autres obstacles propres à la vie en milieu rural, comme le fait de connaître personnellement les fournisseurs de soins ou de suivre des traitements avec d'autres membres de la communauté. Certaines ont dit être très à l'aise dans l'environnement intime et personnel du programme communautaire de lutte contre le cancer, tandis que d'autres ne s'y sentaient pas très bien.
Domaine pratique. L'expérience des participantes était semée de nombreuses embûches pratiques. En effet, la gestion des activités du quotidien n'est pas simple lorsqu'on doit vivre avec des maladies chroniques. De l'aide supplémentaire était aussi requise pour effectuer certaines tâches domestiques ou encore s'occuper d'autres membres de la famille encore dépendants. Certaines femmes disposaient du soutien nécessaire pour y arriver, mais d'autres avaient dû embaucher de l'aide ou se débrouiller seules. Plusieurs devaient souvent s'occuper en plus d'un très grand terrain ou d'une ferme. Le poids des nombreux déplacements, du relogement, des problèmes liés à l'emploi et des pertes financières était des thèmes prédominants de l'étude. Les multiples allers-retours en ville, les conditions routières hasardeuses, les travaux routiers et l'orientation dans une ville inconnue compliquaient aussi l'expérience. Les participantes appréciaient beaucoup que les rendez-vous soient coordonnés avec leurs déplacements, mais les services diagnostiques limités dans les régions rurales venaient alourdir leur horaire.

De plus, les femmes du nord de la province rencontraient des difficultés particulières lorsqu'elles voyageaient en bus ou en avion et, vu leur lieu de résidence, elles étaient forcées de se reloger. Certaines femmes avaient le choix de faire la navette ou de se reloger pour recevoir de la radiothérapie, mais quelle que soit leur décision, elles devaient composer avec le coût du logement ou la fatigue des déplacements. En cas de relogement, l'expérience était plus positive lorsque les participantes trouvaient un logement abordable où elles avaient de l'aide ou, à tout le moins, un endroit confortable. Cependant, toutes n'avaient pas cette chance. Le fardeau financier du cancer était évident. Les difficultés liées à l'emploi, la perte de revenus et les frais à débourser constituaient les principales sources d'inquiétude. L'une des participantes avait des soucis financiers avant le diagnostic et elle a décrit les conséquences dévastatrices de son incapacité à travailler et des coûts qu'entraînait la vie en milieu rural. Elle a fini par déménager pour trouver un logement plus abordable.

Ça m'a coûté cher parce que j'ai dî arrêter de travailler. Je n'avais donc plus de revenus, alors j'ai pas mal épuisé mes économies.

Les frais encourus comprenaient l'essence, le logement, les courses en taxi, les repas, le soutien aux tâches domestiques, le stationnement ou l'achat de fournitures médicales. Bien que les participantes aient apprécié le soutien financier de leurs amis, de leur famille et de leur communauté, les coûts associés à la vie en région demeuraient considérables.

Domaine émotionnel. Pendant les entrevues, les participantes ont évoqué une multitude d'émotions : état de choc, incrédulité, déni, peur, incertitude, colère, culpabilité, sentiments d'anxiété, etc. Ces émotions sont très fréquentes à l'annonce d'un diagnostic de cancer, mais elles sont souvent exacerbées par les conditions de vie en milieu rural à cause du relogement, de la difficulté de traiter avec des fournisseurs de soins inexpérimentés ou insensibles, et des frais supplémentaires à débourser. Le fardeau des déplacements amplifiait aussi le stress émotionnel constant des participantes. L'une d'entre 
elles a raconté avoir dû conduire plusieurs heures pour recevoir les résultats d'une biopsie, habitée pendant tout le trajet d'une grande anxiété. Après une longue attente pour voir le spécialiste, elle est rentrée chez elle, seule avec ses émotions et les nouvelles reçues. Plusieurs histoires semblables ont été rapportées pendant les entrevues.

Ils vont me dire que j'ai le cancer et je dois conduire jusqu'ici, puis attendre deux heures. Ça me mettait en colère.

Plusieurs participantes ont décrit leurs stratégies pour relâcher et gérer les émotions complexes qu'elles ressentaient. Elles trouvaient notamment utile de pleurer, de se défouler ou de s'offrir des moments de solitude. La musique, le yoga, la méditation ou le jardinage les apaisaient également. Plusieurs cherchaient du réconfort auprès de leur famille, leurs amis, leurs collègues, les membres de leur congrégation, les fournisseurs de soins et d'autres survivants du cancer pour les aider à traverser cette épreuve. De plus, les liens communautaires étroits et les soins hautement personnalisés des milieux ruraux de soins du cancer contribuaient aussi à satisfaire leurs besoins émotionnels. La présente étude n’a pas examiné en détail la réaction de la famille à la maladie, mais il semblait évident que l'expérience du cancer est une affaire de famille et que la séparation causée par le relogement ou les déplacements était astreignante sur le plan émotionnel.

Domaine psychologique. Dans ce domaine, les participantes ont rapporté certains besoins non satisfaits, comme la perte de contrôle sur leur vécu en raison de la maladie, la modification de l'image corporelle à cause de la perte de cheveux ou d'un sein, et la perte d'estime de soi. Les femmes devaient composer avec tous ces besoins insatisfaits. Pour certaines, il a été salutaire de reprendre le contrôle en changeant leur perspective sur la maladie ou en se concentrant sur ce que la vie leur apportait de positif. En outre, le fait de se distraire et de vivre la maladie au jour le jour aidait à moins se sentir dépassé. Pour l'une des participantes, le cancer suscitait de nombreuses émotions et la seule manière pour elle de se sentir en contrôle était de vivre dans le moment présent. Dans sa situation, elle avait donc choisi de faire six heures de route par jour pendant ses traitements de radiothérapie, avec le soutien de son conjoint.

J'imagine qu'on s'adapte du mieux qu'on peut. On entre presque dans un état d'esprit qui nous force à agir, à nous concentrer sur ce qu'il faut faire ici et maintenant. Dans mon cas, ce que je devais faire, c'était aller en ville tous les jours.

Il était très important pour les femmes de se sentir bien et de rester en forme; cela renforçait leur sentiment de contrôle et augmentait leur niveau d'énergie. Plusieurs autres caractéristiques, comme la résilience, l'autonomie et l'optimisme, facilitaient l'adaptation et soutenaient l'estime de soi.

Domaine spirituel. L'expérience du cancer, aussi difficile soit-elle, est souvent décrite comme un moment de découverte. Être confrontées à leur propre mortalité s'est révélé très éprouvant pour certaines femmes, mais elles ont, pour la plupart, trouvé des stratégies pour apprivoiser cette incertitude constante. Certaines cherchaient du réconfort dans leurs pratiques spirituelles ou dans la prière, mais elles pouvaient difficilement assister aux offices religieux à cause de contraintes physiques ou sociales. L'une des participantes a dit qu'elle trouvait sa force dans sa foi en Dieu. Elle s'était malgré tout distancée de sa congrégation parce qu'elle avait honte de son diagnostic et qu'elle peinait à en parler.

Je n'aurais pas pu y arriver sans la prière et ma foi en Dieu.

C'est là que je puise toute ma force parce que, seule, je suis

faible et j'ai vraiment dî m'appuyer sur Lui, surtout pendant la chimio.

Certaines femmes ont décrit l'expérience simplement comme un obstacle de plus sur leur chemin. D'autres ne comprenaient pas pourquoi cette situation leur arrivait et ont cherché un sens profond à la maladie. Les participantes ont mentionné l'importance de donner un sens ou de poser un nouveau regard sur l'expérience, par exemple en approfondissant la connaissance de soi, en renforçant les liens familiaux, en étant reconnaissantes d'être en vie et de vivre un jour de plus, en nouant de nouvelles relations et en cultivant celles qui existent déjà. Pour certaines femmes, il s'agissait d'une expérience transformatrice.

\section{DISCUSSION}

La présente étude a permis de préciser l'expérience des soins de soutien pour les femmes des régions rurales du Manitoba qui ont reçu des traitements pour un cancer du sein invasif. Guidées par le cadre de soins de soutien holistique de Fitch (2009), nous avons détaillé tant les aspects positifs que négatifs de la maladie découlant de la vie en milieu rural. Les résultats de l'étude présenteront sans doute un intérêt pour les infirmières qui travaillent en région rurale auprès des patients atteints de cancer. Nos conclusions font écho à celles des autres recherches sur le sujet et peuvent donc servir de canevas à lélaboration d'interventions personnalisées qui amélioreront les soins du cancer offerts aux patients des régions rurales.

Les infirmières en oncologie sont bien placées pour jouer le rôle de chefs de file à différents niveaux du système de santé et pour ouvrir le chemin à l'amélioration des soins prodigués aux patients cancéreux qui vivent en région rurale (Association canadienne des infirmières en oncologie [ACIO], 2001). En ville ou à la campagne, le travail des infirmières est très apprécié; elles se doivent de bien maîtriser les spécificités du monde rural lorsqu'elles soignent des patients cancéreux vivant dans ce milieu.

De plus, les services de navigation étaient considérés comme essentiels pour les femmes qui y avaient recours; ils étaient toutefois sous-utilisés. Les infirmières pivots sont en excellente posture pour offrir des services axés sur les patients et les familles, pour donner de l'enseignement continu, pour défendre les droits des patients et pour servir de lien entre les spécialistes en oncologie, les médecins de première ligne, les fournisseurs de soins du cancer en milieu rural et le personnel en charge des traitements. Elles facilitent aussi la coordination des procédures diagnostiques et des multiples traitements tout en s'occupant de la gestion générale des cas à chaque étape de la trajectoire du cancer (Farber, Deschamps et Cameron, 2002). Cette coordination est déjà essentielle pour les patients atteints de cancer, mais elle est encore plus cruciale pour les 
populations rurales, vu la fragmentation des services. Les concepts d'oncologie ambulatoire, les congés rapides des établissements de soins de courte durée, les plans de traitement complexes et le suivi précoce en milieu de soins de première ligne peuvent dérouter les patients et leur famille. Les infirmières, qu'elles pratiquent dans des établissements voués ou non aux patients cancéreux, peuvent jouer le rôle d'agent de liaison pour assurer la continuité et la coordination des soins tout en tenant compte des réalités complexes de la vie rurale décrites dans la présente étude.

Appliquer les recommandations de la présente étude (Annexe A, tableau 1) permettrait d'améliorer la prestation des services en région et pourrait notamment éviter des retards de diagnostic, raccourcir les délais d'attente, réduire les exigences de déplacement, promouvoir les soins axés sur la famille, améliorer le soutien et les services et faciliter l'adaptation à l'expérience du cancer. Les soins de santé en milieu rural présentent de nombreux défis, déjà abordés dans un rapport commandé par le gouvernement du Manitoba en 2017 (Peachey, Tait, Adams et Croson, 2017). Pour arriver à améliorer les services aux populations rurales, ce rapport recommande, entre autres, une augmentation de l'offre de soins interdisciplinaires dans les milieux de première ligne, la bonification de l'aide à domicile en milieu rural, l'utilisation accrue de la télésanté, et l'augmentation du remboursement des déplacements pour les gens vivant dans les régions géographiquement isolées. À l'opposé, on y souligne qu'une réorganisation et centralisation des services d'urgence et ambulanciers dans la province pourrait se révéler problématique (Peachey, 2017).

De plus, l'objectif stratégique $n^{\circ} 5$ d'Action Cancer Manitoba pour 2016-2021 (ACM, sans date) souligne la nécessité d'améliorer les soins pour les populations mal desservies. La création de nouveaux services et l'amélioration de l'accès pour les résidents des régions isolées ont été désignées comme des priorités. Les auteures du présent article invitent les infirmières du Manitoba et du Canada à prendre connaissance des derniers documents stratégiques du gouvernement et à s'en servir pour provoquer le changement. Les recommandations formulées dans la présente étude ne sont pas nouvelles, mais elles demeurent valables et devraient être prioritaires.

Létude présente certaines limites, qu'il importe de mentionner. D'abord, la différence d'âge entre les participantes n'a pas été examinée en profondeur. Pendant les entrevues, l'âge ne semblait pas avoir une si grande influence sur l'expérience du cancer. Néanmoins, la question du lien entre l'âge et l'expérience du cancer du sein mérite sans conteste d'être explorée davantage. De plus, les personnes vivant en région isolée n'étaient pas représentées dans l'étude. On peut toutefois présumer que cela exacerbe encore plus les défis à surmonter. En outre, plusieurs thèmes sont absents de l'étude, notamment les hommes vivant avec une maladie, le bagage ethnoculturel, les survivants à long terme, le diagnostic de maladie avancée ou de stade palliatif et les proches aidants. Ces éléments devraient faire l'objet de futures recherches afin de mieux comprendre toutes les nuances de l'expérience rurale chez différentes populations.

Les résultats de la présente recherche ont tout de même produit de riches descriptions et ouvert de nouvelles perspectives sur l'expérience de la ruralité tout en faisant la lumière sur les domaines où un changement est souhaitable, voire nécessaire. Tous devront coopérer pour donner suite aux témoignages des femmes et concrétiser l'objectif d'amélioration du soutien et des services. Les infirmières, en collaboration avec les autres professionnels de la santé qui travaillent avec les patients cancéreux des régions rurales, sont bien placées pour être la figure de proue de ce mouvement.

\section{CONCLUSION}

Létude décrit les besoins en soins de soutien des femmes des régions rurales atteintes de cancer du sein, du diagnostic à la fin du traitement. Les résultats et les recommandations de l'étude doivent être envisagés dans une perspective nationale, puisque l'application de modèles de services aux patients atteints de cancer vivant en région isolée constitue un défi partout au pays. Nous ne pouvons pas changer le fait qu'il y a des populations rurales au Canada et que les services spécialisés en oncologie sont principalement offerts dans les grandes villes. Toutefois, les infirmières et les autres fournisseurs de soins doivent tenir compte des caractéristiques et conditions particulières des patients des régions rurales qui reçoivent un diagnostic de cancer. Il reste beaucoup à faire pour améliorer le soutien et les services, de même que la qualité de vie et les soins des populations rurales aux prises avec cette terrible maladie. Les résultats de la présente étude explorent ce sujet si important et serviront de point de départ à la prise de conscience et au changement.

\section{ANNEXE A}

\section{Tableau 1. Recommandations pour la prestation des services}

- Augmenter le nombre de procédures diagnostiques dans les milieux ruraux.

- Encourager les spécialistes à se rendre dans les collectivités rurales.

- Enregistrer les séances avec les spécialistes.

- Améliorer la connaissance et l'utilisation des programmes de navigation.

- Améliorer les services de première ligne en milieu rural et les liens avec ACM.

- Accroître le recours à des équipes de soins interdisciplinaires dans les régions rurales.

- Recourir davantage à la télésanté.

- Améliorer les soins d'urgence en milieu rural.

- Encourager la présence d'un membre de la famille pour les déplacements afin de favoriser la proche aidance.

- Prévoir le moment du congé et les rendez-vous en fonction des déplacements.

- Promouvoir des services de soutien à orientation sociale, comme les services de repas et de transport, ou alors les programmes d'éducation ou de soutien par les pairs en milieu rural.

- Augmenter le nombre d'installations d'aide au relogement et de logements en milieu urbain.

- Améliorer l'esthétique des établissements ruraux de soins du cancer et y aménager des espaces privés.

- Multiplier les occasions de formation en oncologie pour les généralistes en exercice. 


\section{RÉFÉRENCES}

Action Cancer Manitoba [ACM] (2000). CCP program directory. [En ligne] http://www.cancercare.mb.ca/home/patients_and_ family/treatment_services/treating_patients_in_rural_manitoba/ rural_program_directory/

Action Cancer Manitoba [ACM] (2017). Current programs. [En ligne] http://www.cancercare.mb.ca/home/patients_and_family/patient_ and_family_support_services/current_programs/index.cfm

Action Cancer Manitoba [ACM] (s.d.) Delivering excellence: 20162021 Manitoba cancer plan. [En ligne] https://www.cancercare. mb.ca/export/sites/default/About-Us/.galleries/files/corporatepublications/Manitoba_Cancer_Plan_2016-2021.pdf

Adams, P., Hardwick, J., Embree, V., Sinclair, S., Conn, B. et Bishop, J. (2009). Models of cancer services for rural and remote communities. [En ligne] http://www.cancerinstitute.org.au/media/70218/web0983-02_literature_review_models_cancer_services_rural_and_ remote_communities.pdf

Association canadienne des infirmières en oncologie (ACIO) (2001). Standards of care, roles in oncology nursing, and role competencies. [En ligne] http://www.cano-acio.ca/ ASSETS/DOCUMENT/ Practice/standards\%20of\%20care.pdf

Carlow, D.R. (2000). The British Columbia Cancer Agency: A comprehensive and integrated system of cancer control. Health Quarterly, 3(3), 31-45. doi:10.12927/hcq.16755.

Clinical Oncological Society of Australia (2006). Mapping rural and regional oncology services in Australia. [En ligne] https://www.cosa. org.au/media/1067/cosa_paper_mapping-regional-oncologyservices_2006.pdf

Compas, B.E. et Luecken, L. (2002). Psychological adjustment to breast cancer. American Psychological Society, 11(3), 111-114.

Crane-Okada, R. et Loney, M. (2007). Breast cancer. Dans M.E. Langhorne, J.S. Fulton et S.E. Otto (éditeurs), Oncology nursing (5 éd., p. 101-124). St. Louis: Mosby Elsevier.

Farber, J.M., Deschamps, M. et Cameron, R. (2002). Investigation and assessment of the navigator role in meeting the informational, decisional and educational needs of women with breast cancer in Canada. [En ligne] http://publications.gc.ca/collections/Collection/H39663-2002E.pdf

Fitch, M.I., Gray, R.E., McGowan, T., Bunskill, I., Steggles, S., Sellick, S., Bezjak, A. et McLeese, D. (2003). Traveling for radiation cancer treatment: Patient perspectives. Psycho-Oncology 12, 664674. doi:10.1002/pon.682.

Fitch, M.I. (2009). Supportive care framework: Theoretical underpinnings (1 ${ }^{\mathrm{re}}$ éd.). Dans M.I. Fitch, H.B. Porter et B.D. Page (éditeurs), Supportive care framework: A foundation for personcentred care (p. 11-29). Pembroke, ON: Pappin Communications.

Gray, R.E., James, P., Manthorne, J., Gould, J. et Fitch, M.I. (2004). A consultation with Canadian rural women with breast cancer. Health Expectations, 7, 44-50.
Loughery, J. et Woodgate, R.L. (2015). The supportive care needs of rural individuals living with cancer: A literature review. Canadian Oncology Nursing Journal, 25(2), 157-166. doi:10:5737/23688076252157166

Mathews, M., West, R. et Buehler, S. (2009). How important are outof-pocket costs to rural patients' cancer care decisions? Canadian Journal of Rural Medicine, 14(2), 54-60.

National Comprehensive Cancer Network (2014). Breast cancer. [En ligne] http://www.nccn.org/professionals/physician_gls/pdf/ breast.pdf

National Institute for Clinical Excellence (2004). Guidance on cancer services: Improving supportive and palliative care for adults with cancer. [En ligne] http://www.nice.org.uk/guidance/csgsp/ evidence/supportive-and-palliative-care-the-manual-2

Peachey, D., Tait, N., Adams, O. et Croson, W. (2017). Provincial clinical and preventative services planning for Manitoba: Doing things differently and better. [En ligne] https://www.gov.mb.ca/health/ documents/pcpsp.pdf

Pesut, B., Robinson, C.A., Bottorff, J.L., Flyles, G. et Brougton, S. (2010). On the road again: Patients' perspectives on commuting for palliative care. Palliative and Supportive Care, 8, 187-195. doi:10.1017/S1478951509990940.x.

Rogers-Clark, C. (2002). Living with breast cancer: The influence of rurality on women's suffering and resilience. A postmodern feminist inquiry. Australian Journal of Advanced Nursing, 20(2), 34-39.

Société canadienne du cancer [SCC] (2017). Canadian cancer statistics 2017: Special topic: Pancreatic cancer. [En ligne] http://www.cancer. $\mathrm{ca} / \sim /$ media/cancer.ca/CW/cancer\%20information/cancer\%20 101/Canadian\%20cancer\%20statistics/Canadian-Cancer-Statistics2017-EN.pdf?la=en

Statistique Canada (2015). Canada's rural population since 1851. [En ligne] http://www12.statcan.gc.ca/census-recensement/2011/ as-sa/98-310-x/98-310-x2011003_2-eng.cfm

Streubert, H. et Carpenter, R.D. (2011). Qualitative research in nursing: Advancing the humanistic imperative. $5^{\mathrm{e}}$ éd. Philadelphia: Lippincott Williams \& Wilkins.

Thorne, S. (2008). Interpretive description. Walnut Creek, CA: Left Coast Press.

Winter, C.A. et Lee, H.J. (2010). Rural nursing: Concepts, theory and practice ( $3^{\mathrm{e}}$ éd.) Springers Publishing Company, LLC: New York.

Zhang, Y. et Wildemuth, B.M. (2009). Qualitative analysis of content. In B. Wildemuth (éd.) Applications of social research methods to questions in information and library science, p. 308-391. Westport, CT; Library Limited. 